\title{
Sudden Death in Middle Aged Woman with Invasive Pulmonary Aspergillosis
}

\section{Ahmed Fayed*}

Department of Internal Medicine, King Abdul Aziz Specialized Hospital, Tai'f SKA, Saudi Arabia

\begin{abstract}
A 45-year-old Saudi female with history of SLE on hydroxychloroquine $400 \mathrm{mg}$ daily and prednisolone $5 \mathrm{mg}$ daily Presented to ER by epigastric pain, diarrhoea 3 motions per day and generalized body aches associated with infrequent cough for past 2 days not preceded by flu symptoms without contact to sick people for last month, without history of recent travel and without contact to animals.
\end{abstract}

Keywords: Pulmonary aspergillosis; Pneumonia; Coughing

\section{Case Report}

In ER patient was Conscious, oriented, cooperative but appear ill with temp $38^{\circ} \mathrm{C}$, HR 100 beat/minute, RR16 cycle/minute, Bp 110/70 $\mathrm{mmHg}$ and $\mathrm{SO}_{2} 81 \%$ RA Chest examination unremarkable abdominal examination lax and soft without tenderness or palpable mass or organomegally lab WBC $0.6 \mathrm{Hb} 16.4$ Plt 85 Cr 1.2 sGOT 1400 sGPT 340 lipase 476 amylase $124 \mathrm{LDH} 1700$ (Figure 1). So patient admitted in ICU isolation started on ceftriaxonem, azithromycin, ostlmivir, steroid pulse therapy and other supportive measures with swabbing for seasonal influenza and MERS-cov which came later + ve for influenza A and ECHO done with normal study.

\section{Through next week}

Throat swab revealed klebsiella pneumonia and chest examination right sided crepitation MRSA -ve, blood culture -ve, urine culture -ve, salmonella and brucella -ve, normal level of C3 and C4, -ve HCV, -ve HBV, -ve HIV (asked by infectious disease team), -ve rheumatoid factor, -ve anti ds DNA, -ve anticardiolipin antibody (asked by rheumatologist) CBC showed steady decreased in HB 12.8 and improved WBC 5.6 but decreased Plt 44 so occult blood in stool requested by gastrologist and reported -ve with partial improvement in liver function sGOT 400 sGPT 200 and preserved renal function (Figures 2 and 3).

\section{At $7^{\text {th }}$ day}

Patient deteriorate and put on NIMV although lab panel improved (WBC $6.4 \mathrm{Hb} 11$ Plt 70 sGPT 42 sGOT 49 alb 2.5 Cr 1.2 LDH 500) with coughing of brownish plug in night while off of NIMV (Figures 4 and 5).

More deterioration so intubated with mech. Ventilation and sputum culture +ve for heavy aspergillus growth and diagnosis of invasive pulmonary aspergllosis established with antifungal started After this patient progress smoothly with regression of fever and improvement
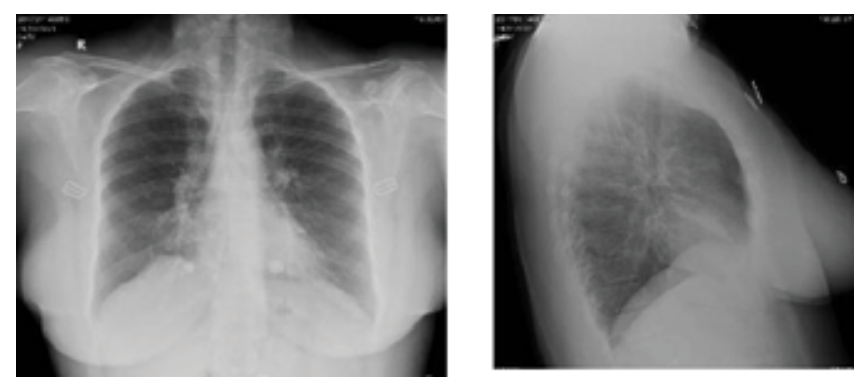

Figure 1: Chest X-ray heterogeneous opacity obliterating right heart border with right diaphragmatic hump.

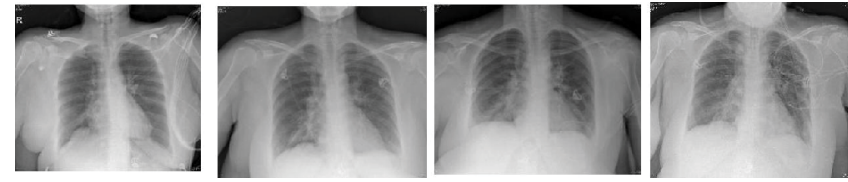

Figure 2: Serial CXR show increased in opacity on right side.

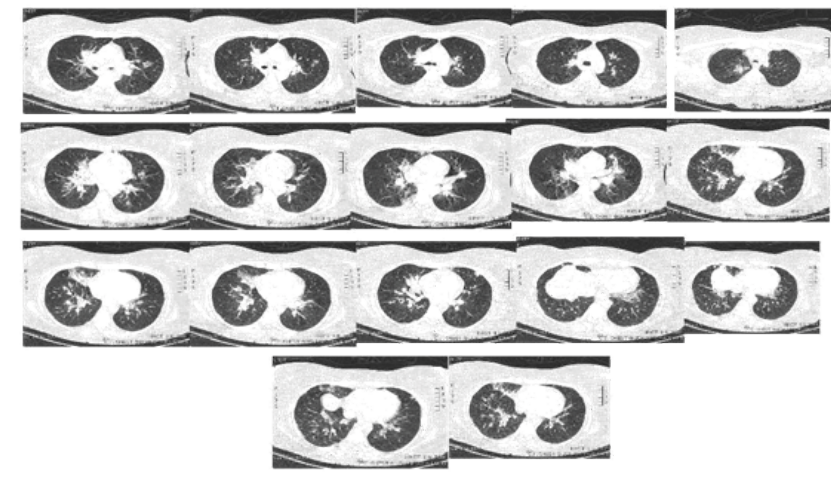

Figure 3: HRCT chest.

of ventilator parameters and all lab normalized except for $\mathrm{Hb}$ decrease with again -ve occult blood in stool and with MRI brain reported as right fronto-parietal lesions with double hallo sign mostly of infectious origin tubeculoma or small abscesses and chest $\mathrm{X}$-ray gradually improved (Figure 6).

\section{At $12^{\text {th }}$ day}

Antibiotics (imepenem, moxifloxacin and vancomycin), ostlmivir and steroid stopped and antifungal with ostlmivir continued by committee of consultant rheumatologist, pulmonologist and infectious

*Corresponding author: Ahmed Fayed, Department of Internal Medicine, King Abdul Aziz Specialized Hospital, Tai'f SKA, Saudi Arabia, Tel: 966594203755 E-mail: chestradiology@yahoo.com

Received January 04, 2018; Accepted January 11, 2018; Published January 18 2018

Citation: Fayed A (2018) Sudden Death in Middle Aged Woman with Invasive Pulmonary Aspergillosis. J Pulm Respir Med 8: 441. doi: 10.4172/2161105X.1000441

Copyright: (c) 2018 Fayed A. This is an open-access article distributed under the terms of the Creative Commons Attribution License, which permits unrestricted use, distribution, and reproduction in any medium, provided the original author and source are credited. 


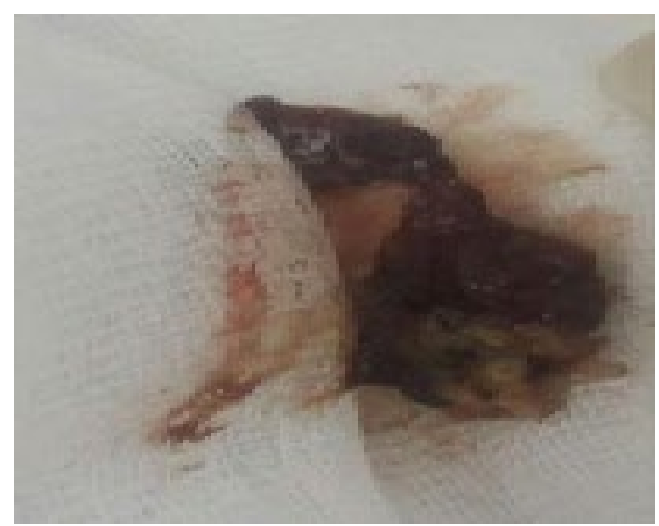

Figure 4: Brownish plug in night while off of NIMV.

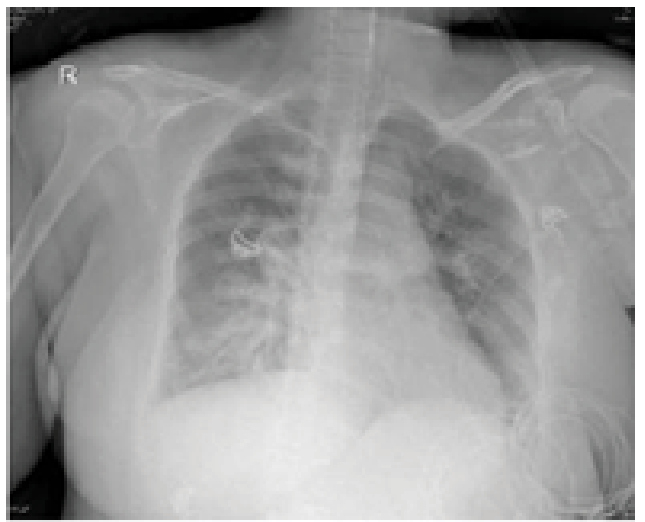

Figure 5: Chest X-ray show increased infiltration on right side with some less infiltration on left side.
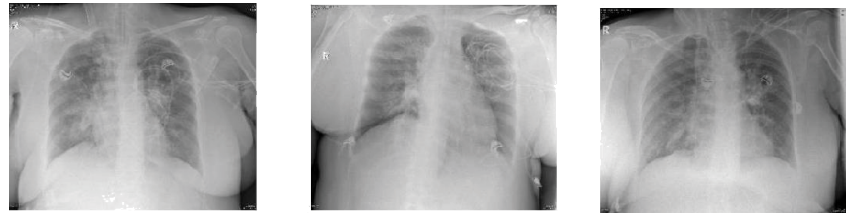

Figure 6: CT abdomen reported as bilateral calcified gluteal nodules with right phlebolith with lower chest cuts showed patches of consolidation more on right.

disease but weaning was difficult possibly from steroid induced myopathy.

\section{2 days later}

Patient developed fever with increased CXR infilteration figure so diagnosis of VAP established and antibiotics started again (linezolid, imepenem and colistin) with septic screen request and chest X-ray showed right lower zone heterogeneous opacity (Figure 7).

\section{Next 2 days}

Urine culture revealed klebsiella pneumonia sensitive to linezolid and imepenem so colistin stopped And patient swabbed again for H1N1 and MERS-COV (came later -ve) and patient look hopeful without any inotropic support or sedation with $\mathrm{FiO}_{2} 40 \%$ PEEP 5 PS 12 and $\mathrm{SO}_{2}$ was 97\%, HR 88, temp $36.8^{\circ} \mathrm{C} \mathrm{Bp} 130 / 80 \mathrm{mmHg}$ with chest X-ray less better

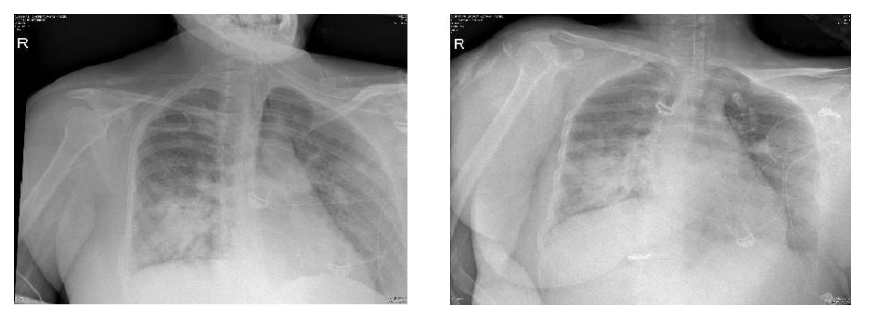

Figure 7: Chest X-ray showing right lower zone heterogeneous opacity.

(Figure 8) and weaning trial adviced but unfortunately sudden attack of massive hemoptysis at PM shift with about $1200 \mathrm{ml}$ fresh blood from ETT patient arrested and not revive.

\section{Case Review}

Aspergillus species are ubiquitous in nature and can affect lung in many ways; Allergic bronchopulmonary aspergillosis, invasive aspergillosis, subacute invasive pulmonary aspergillosis and chronic pulmonary aspergillosis [1].

\section{Invasive pulmonary aspergillosis}

Inhalation of infectious conidia is a frequent event but tissue invasion is uncommon occurring most frequently in the setting of immunosuppression including steroid therapy [2] and when there is a high amount of airway exposure as in the setting of construction [3].

While invasive aspergillosis most commonly involves the lungs it can disseminate beyond the respiratory tract to multiple different organs, including the skin, brain, eyes, liver, and kidneys and most commonly are caused by A. fumigatus complex, followed by A. flavus, A. niger, and A. terreus [4].

Patients can present with fever, chest pain, shortness of breath, cough, and/or hemoptysis and the classic triad that has been described in neutropenic patients with pulmonary aspergillosis is fever, pleuritic chest pain, and hemoptysis [1].

Invasive pulmonary aspergillosis typically manifests as single or multiple nodules with or without cavitation, patchy or segmental consolidation, or peribronchial infiltrates with or without tree-in-bud patterns [5].

Optimal management involves early diagnosis and early initiation of antifungal therapy and the duration of antifungal therapy is dependent upon the location of the infection, the patient's underlying disease, the need for further immunosuppression, and the response to therapy with the minimum duration of therapy is 6 to 12 weeks [6].

While three classes of antifungal agents are available for the treatment of aspergillosis: polyenes (amphotericin B lipid formulation), azoles (voriconazole, isavuconazole, posaconazol), and echinocandins (caspofungin, micafungin, and anidulafungin), Voriconazole is the main therapy for most patients and the preferred monotherapy by most of exper but For initial therapy of severe invasive aspergillosis combination therapy of voriconazole and an echinocandin is recommended and For patients who are intolerant of voriconazole due to severe reactions, a lipid formulation of amphotericin B or isavuconazole is recommended. In patients with refractory or progressive invasive aspergillosis altering the antifungal regimen (ideally changing the class of antifungal), reducing immunosuppression when feasible and surgical resection of necrotic lesions in some cases should be considered [6,7]. 


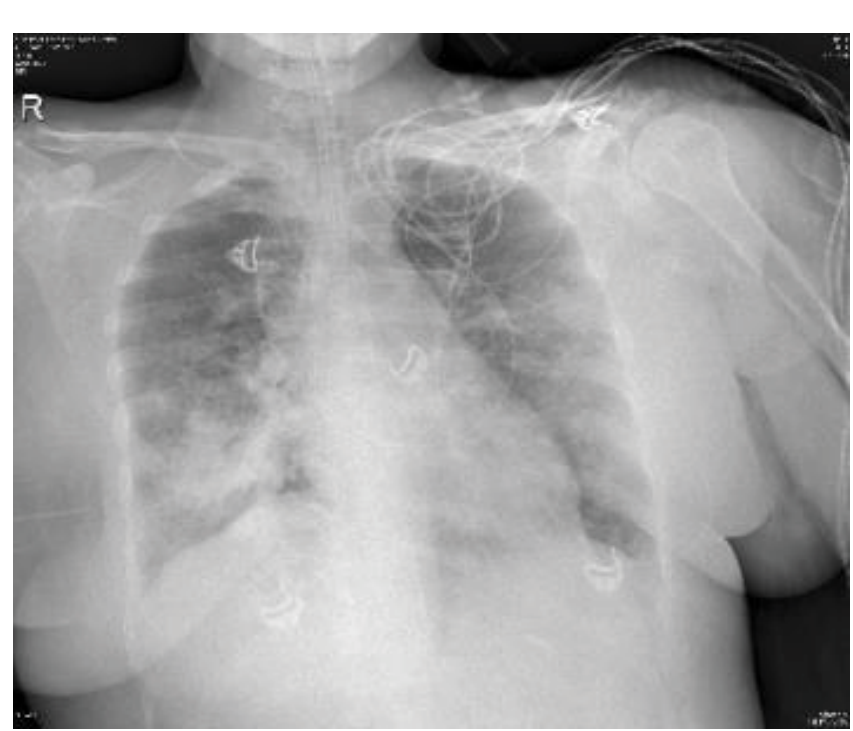

Figure 8: Chest X-ray of patient on inotropic support.

\section{Allergic Bronchopulmonary Aspergillosis (ABPA)}

It is a complex hypersensitivity reaction in response to colonization of the airways with Aspergillus fumigatus that occurs almost exclusively in patients with asthma or cystic fibrosis [8] characterized pathologically by mucoid impaction of the bronchi, eosinophilic pneumonia, and bronchocentric granulomatosis [9].

Diagnostic criteria include positive Aspergillus skin test or detectable IgE levels against Aspergillus fumigatus, elevated total blood eosinophil count (generally $>500$ cells/microL), elevated total serum IgE (generally $>1000 \mathrm{IU} / \mathrm{mL}$ ), precipitating IgG antibodies (precipitins) to Aspergillus, and also specific $\operatorname{IgE}$ and $\operatorname{IgG}$ antibodies to Aspergillus on immunoassay, central bronchiectasis, Expectoration of goldenbrown plugs in the sputum [10-12]. While Oral corticosteroids are the mainstay for the treatment of ABPA, Itraconazole may be added in patients experiencing recurrent exacerbations despite adequate steroid therapy but. The role of itraconazole monotherapy for ABPA is still not established and omalizumab could possibly have steroid-sparing alternative [13].

\section{Chronic pulmonary aspergillosis}

Includes several disease manifestations including aspergilloma, aspergillus nodules, chronic cavitary pulmonary aspergillosis and subacute invasive pulmonary aspergillosis and Almost all cases of chronic pulmonary aspergillosis are caused by Aspergillus fumigatus, although patients have been described with $A$. niger or $A$. flavus infection $[1,4]$.

All patients with chronic pulmonary aspergillosis have either prior pulmonary damage or disease Such as pulmonary tuberculosis, nontuberculous mycobacterial infection, allergic bronchopulmonary aspergillosis, lung cancer (following successful treatment), prior pneumothorax with associated bulla formation, chronic obstructive pulmonary disease, and fibrocavitary sarcoidosis [14-16].

The cardinal test for chronic pulmonary aspergillosis is a positive aspergillus immunoglobulin (IgG antibody test from the serum. Elevated inflammatory markers, such as C-reactive protein and/or erythrocyte sedimentation rate, are very common in patients with chronic pulmonary aspergillosis but are not specific [17].

\section{Subacute invasive pulmonary aspergillosis (Chronic necrotizing pulmonary aspergillosis)}

Occurs in immune compromised patients with hyphal invasion of tissue is observed histologically and progressive features over one to three months. This manifestation typically occurs in patients with diabetes mellitus, malnutrition, alcoholism, advanced age, prolonged glucocorticoid use or other modestly immunosuppressive agents, chronic obstructive pulmonary disease, connective disease, radiation therapy, non-tuberculous mycobacterial infection, or Human Immunodeficiency Virus (HIV) infection [1]. Such patients usually have a single thin-walled cavity or area of cavitating pneumonia/consolidation and may have detectable aspergillus antigen (galactomannan) or aspergillus IgG antibodies in blood [6].

\section{References}

1. Denning DW, Cadranel J, Beigelman-Aubry C (2016) Chronic pulmonary aspergillosis: Rationale and clinical guidelines for diagnosis and management. Eur Respir J 47: 45.

2. Meersseman W, Lagrou K, Maertens J, Van Wijngaerden E (2007) Invasive aspergillosis in the intensive care unit. Clin Infect Dis 45: 205.

3. Kanamori H, Rutala WA, Sickbert-Bennett EE, Weber DJ (2015) Review of fungal outbreaks and infection prevention in healthcare settings during construction and renovation. Clin Infect Dis 61: 433.

4. Balajee SA, Marr KA (2006) Phenotypic and genotypic identification of human pathogenic aspergilli. Future Microbiol 1: 435.

5. Horger M, Hebart H, Einsele $\mathrm{H}$ (2005) Initial CT manifestations of invasive pulmonary aspergillosis in 45 non HIV immunocompromised patients: Association with patient outcome? Eur J Radiol 55: 437.

6. Patterson TF, Thompson GR, Denning DW (2016) Practice guidelines for the diagnosis and management of aspergillosis: 2016 update by the infectious diseases society of america. Clin Infect Dis 63: e1.

7. Marr KA, Schlamm HT, Herbrecht R (2015) Combination antifungal therapy for invasive aspergillosis: A randomized trial. Ann Intern Med 162: 81

8. Agarwal R (2009) Allergic bronchopulmonary aspergillosis. Chest 135: 805 826.

9. Iscili BP, Wood KL (2009) Noninvasive pulmonary Aspergillus infections. Clin Chest Med 30: 315.

10. Agarwal R, Chakrabarti A, Shah A (2013) Allergic bronchopulmonary aspergillosis: Review of literature and proposal of new diagnostic and classification criteria. Clin Exp Allergy 43: 850.

11. Buckingham SJ, Hansell DM (2003) Aspergillus in the lung: Diverse and coincident forms. Eur Radiol 13: 1786.

12. Ashok S, Chandramani $P$ (2014) Allergic aspergillosis of the respiratory tract Eur Respir Rev 23: 8-29.

13. Fink JN (2000) Therapy of allergic bronchopulmonary aspergillosis. Indian J Chest Dis Allied Sci 42: 221-224.

14. Sonnenberg P, Murray J, Glynn JR (2000) Risk factors for pulmonary disease due to culture-positive $M$. tuberculosis or nontuberculous mycobacteria in South African gold miners. Eur Respir J 15: 291.

15. Lee JJ, Chong PY, Lin CB (2008) High resolution chest CT in patients with pulmonary tuberculosis: Characteristic findings before and after antituberculous therapy. Eur J Radiol 67: 100.

16. Aspergilloma and residual tuberculous cavities-the results of a resurvey Tubercle 51: 227.

17. Denning DW, Riniotis K, Dobrashian R, Sambatakou H (2003) Chronic cavitary and fibrosing pulmonary and pleural aspergillosis: Case series, proposed nomenclature change, and review. Clin Infect Dis 37 Suppl 3: S265. 